\title{
Abundances in extremely metal-poor stars: comparison of the trends of abundance ratios in giants and turnoff stars
}

\author{
Monique Spite ${ }^{1}$, Piercarlo Bonifacio ${ }^{1},{\text { Roger } \text { Cayrel }^{1} \text {, François Spite }}^{1}$, \\ Vanessa Hill ${ }^{1}$, Johannes Andersen ${ }^{2}$, Beatriz Barbuy ${ }^{3}$, Timothy \\ Beers $^{4}$, Eric Depagne ${ }^{5}$, Patrick François ${ }^{5}$, Paolo Molaro ${ }^{6}$, Birgitta \\ Nordström ${ }^{2,7}$, Bertrand Plez ${ }^{8}$, and Francesca Primas ${ }^{9}$ \\ ${ }^{1}$ GEPI, Observatoire de Paris-Meudon, F-92125 Meudon Cedex, France, \\ ${ }^{2}$ Astron. Obs., NBIfAFG, Juliane Maries Vej 30, DK-2100 Copenhagen, Denmark, \\ ${ }^{3}$ IAG, Universidade de Sao Paulo, Depto. de Astronomia, rua do Matao 1226, Sao Paulo \\ 05508-900, Brazil, \\ ${ }^{4}$ Department of Physics \& Astronomy and JINA: Joint Institute for Nuclear Astrophysics, \\ Michigan State University, East Lansing, MI 48824, USA, \\ ${ }^{5}$ ESO, 3107 Alonso de Cordova, Vitacura, Casilla 19001, Santiago 19, Chile, \\ ${ }^{6}$ Osservatorio Astronomico di Trieste, INAF, Via G.B. Tiepolo 11, I-34131 Trieste, Italy, \\ ${ }^{7}$ Lund Observatory, Box 43, SE-221 00 Lund, Sweden \\ ${ }^{8}$ GRAAL, Université de Montpellier II, F-34095 Montpellier Cedex 05, France, \\ ${ }^{9}$ ESO, Karl Schwarzschild-Str. 2, D-85749 Garching b. München, Germany,
}

\begin{abstract}
We show that in a sample of Extremely Metal-Poor (EMP) giants $(<[\mathrm{Fe} / \mathrm{H}]\rangle=$ -3.1 ) all the stars with a luminosity higher than $\log \mathrm{L} / \mathrm{L} \odot \approx 2.6$ present the characteristics of a mixing of the surface with the H-burning layer: low abundance of carbon and high abundance of nitrogen. In these "mixed stars" the lithium abundance and the ratio ${ }^{12} \mathrm{C} /{ }^{13} \mathrm{C}$ are very low. Some of these stars are also $\mathrm{Na}$ or/and $\mathrm{Al}$ rich.
\end{abstract}

Keywords. Stars: abundances, stars: atmospheres, stars: Population II, subdwarfs, supernovae, nucleosynthesis

\section{Introduction}

The aim of the ESO Large Program "First Stars" was to obtain a precise determination of the chemical composition of the Galactic matter in the early stages of the Galactic evolution. These abundances indeed reflect the yields of the first supernovae and provide useful constraints on their models.

As a first approximation the chemical composition of a star does not change during its life and thus the chemical composition of the atmosphere of the very old galactic stars can be considered as a good witness of the Galactic matter in the early times. However if during the life of the star a mixing happens between the surface of the star and the deep layers where nucleosynthetic processes take place, this hypothesis is no more valid and the abundances measured in the stellar atmospheres are not representative of the abundances in the pristine gas. Since in extremely metal-poor stars (EMP) many elements can be measured only in giant stars, it is important to check wether a deep convection has altered the original chemical composition of the atmosphere of the star. 


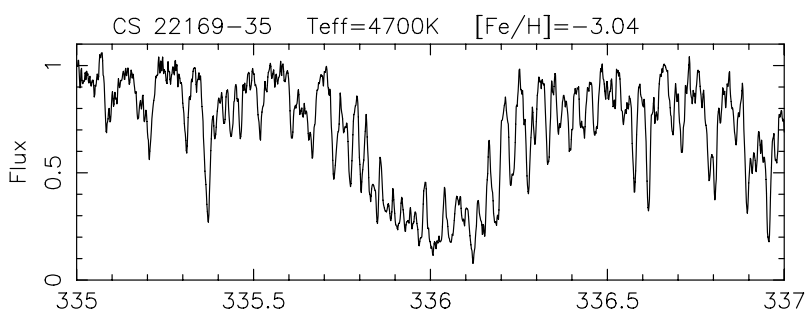

Figure 1. Example of a spectrum in the region of the $\mathrm{NH}$ band at $336 \mathrm{~nm}$
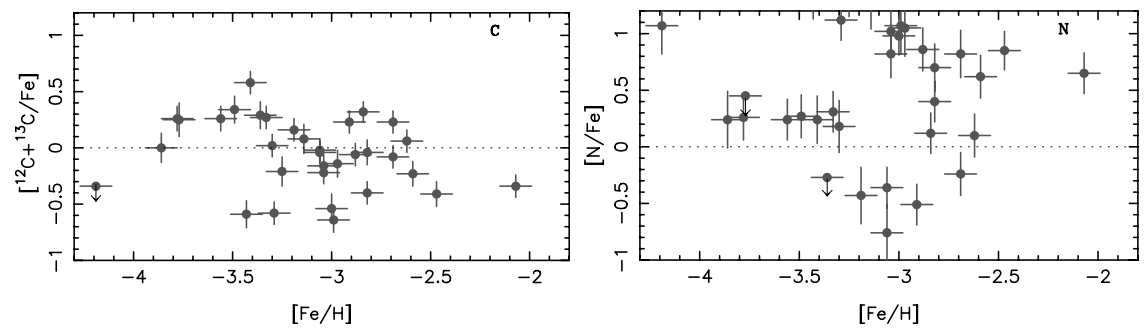

Figure 2. In the EMP giant stars, the scatter of $[\mathrm{C} / \mathrm{Fe}]$ and $[\mathrm{N} / \mathrm{Fe}]$ at low metallicity is very large unlike the other elements like $\mathrm{Mg}$, Ca etc...

\section{Observations and analysis}

Our sample of extremely metal-poor stars was selected in the catalogue of Beers, Preston and Schectman 1992. It now includes a total of 52 normal EMP stars: 33 giants and 18 turnoff stars $(33$ of them with $[\mathrm{Fe} / \mathrm{H}]<-3.0$. This paper deals only with the sample of EMP giants. All these stars are "normal metal-poor stars" i.e. not carbon-rich: $[\mathrm{C} / \mathrm{Fe}]<+0.8$. (The carbon-rich stars are a peculiar class of stars which represents about $25 \%$ of the EMP stars).

The observations were performed at a resolving power of $\mathrm{R}=47000$ at $340 \mathrm{~nm}$ and taking advantage of the high efficiency of UVES in the UV region, we could measure the nitrogen abundance from the $\mathrm{NH}$ band at $336 \mathrm{~nm}$ in most of our giants (Fig. 1).

Our sample of giants is the largest sample of EMP giants in which the abundance of $\mathrm{C}$ and $\mathrm{N}$ has been determined. Some years ago, a similar work has been done by Gratton et al. (2000) at a lower deficiency: $[\mathrm{Fe} / \mathrm{H}]=-1.5$. The comparison of both samples will provide the variation of the mixing phenomenon with metallicity.

We carried out a classical LTE analysis using OSMARCS models (Plez et al. 1992, Edvardsson et al. 1993), the abundances were derived using the current version of the Turbospectrum code (Alvarez \& Plez, 1998). The temperatures were deduced from the color indices calibrated by the IRFM method (Alonso et al. 1999) and the gravity from the ionisation equilibrium.

\section{Abundances of $\mathrm{C}$ and $\mathrm{N}$}

For the elements "X" we have computed the ratio $[\mathrm{X} / \mathrm{Fe}]$. Generally speaking, when $[\mathrm{X} / \mathrm{Fe}]$ is plotted versus $[\mathrm{Fe} / \mathrm{H}]$ in the interval $-4<[\mathrm{Fe} / \mathrm{H}]<-3$, the ratio $[\mathrm{X} / \mathrm{Fe}]$ is constant and the scatter is small: $\sigma \approx 0.1$ dex.

However $\mathrm{C}$ and $\mathrm{N}$ make an exception. The carbon abundance $\left({ }^{12} \mathrm{C}\right.$ and $\left.{ }^{13} \mathrm{C}\right)$ is determined from the profile of the $\mathrm{G}$ band of $\mathrm{CH}$ at $430 \mathrm{~nm}$ and the nitrogen abundance from the $\mathrm{NH}$ band at $336 \mathrm{~nm}$. 


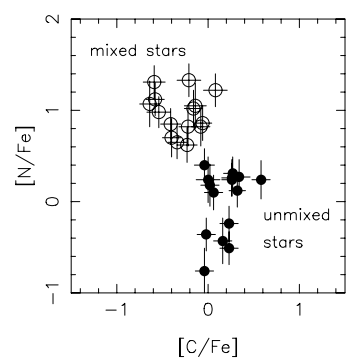

Figure 3. $[\mathrm{N} / \mathrm{Fe}]$ is correlated with $[\mathrm{C} / \mathrm{Fe}]$ in our sample of EMP giants. Two groups of stars can be defined: the "mixed" (open circles) and the "unmixed" giants (filled circles).
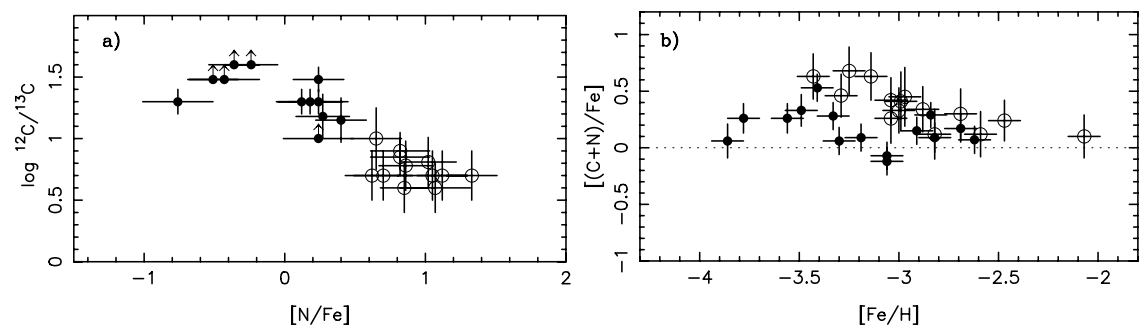

Figure 4. The symbols are the same as in Fig. 3. -a) In the EMP giants there is a good correlation between ${ }^{12} \mathrm{C} /{ }^{13} \mathrm{C}$ and $[\mathrm{N} / \mathrm{Fe}]$. -b) The total abundance of $\mathrm{C}+\mathrm{N}$ is very similar in the mixed and unmixed stars. The slight difference can be generally explained by the errors.

When $[\mathrm{C} / \mathrm{Fe}]$ or $[\mathrm{N} / \mathrm{Fe}]$ are plotted versus $[\mathrm{Fe} / \mathrm{H}]$ the scatter is very large: $\sigma(\mathrm{C})=0.31 \mathrm{dex}$ and $\sigma(\mathrm{N})=0.57$ dex, see Fig. 2 .

There are two possible explanations: either this dispersion represents a real dispersion of $\mathrm{C}$ and $\mathrm{N}$ in the interstellar medium of the early Galaxy (primordial scenario) or the original $\mathrm{C}$ and $\mathrm{N}$ abundances in the atmosphere of the giant stars have been altered by a variable mixing with the $\mathrm{H}$ burning layer.

\section{Consequences of a mixing of the surface with the $\mathbf{H}$ burning layer}

\subsection{Abundances of $C$ of $N$ and the ${ }^{12} \mathrm{C} /{ }^{13} \mathrm{C}$ ratio}

In the $\mathrm{H}$ burning layer, the $\mathrm{CN}$ process is active: ${ }^{12} \mathrm{C}$ is transformed into ${ }^{13} \mathrm{C}$ and ${ }^{13} \mathrm{C}$ into ${ }^{14} \mathrm{~N}$. If the surface of the star is mixed to this layer, little by little the abundance of ${ }^{13} \mathrm{C}$ and of ${ }^{14} \mathrm{~N}$ increases.

If the large scatter of the abundance of $\mathrm{C}$ and $\mathrm{N}$ observed in our sample of giants is due to a mixing with the $\mathrm{H}$ burning layer then we expect:

1- an anticorrelation between $[\mathrm{C} / \mathrm{Fe}]$ and $[\mathrm{N} / \mathrm{Fe}]$

2- a high value of the ${ }^{13} \mathrm{C}$ abundance in the nitrogen-rich (and thus carbon-poor) stars and thus a low value of the ratio ${ }^{12} \mathrm{C} /{ }^{13} \mathrm{C}$

3- that the total number of atoms $\mathrm{C}+\mathrm{N}$ be about the same in all the stars.

These propositions are illustrated by figures $3,4.1 \mathrm{a}$ and $4.1 \mathrm{~b}$ which are all in favor of a mixing explanation.

Fig. 3 shows the correlation between $[\mathrm{C} / \mathrm{Fe}]$ and $[\mathrm{N} / \mathrm{Fe}]$. We can define two groups of giants : a group of nitrogen-rich and carbon-poor stars (called hereafter "mixed stars") and a second group which is carbon-rich but nitrogen-poor (called hereafter "unmixed stars"). 


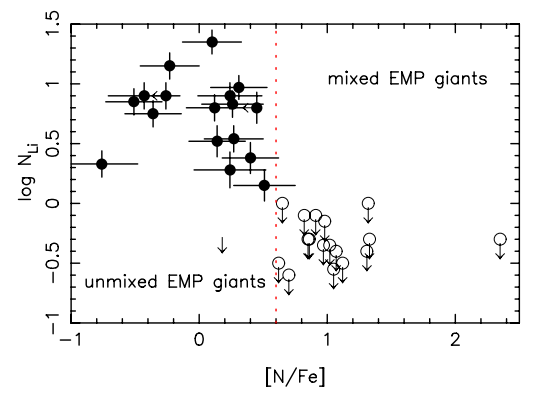

Figure 5. The symbols are the same as in Fig. 3. The lithium abundance is anticorrelated with the nitrogen abundance. In the mixed stars $([\mathrm{N} / \mathrm{Fe}]>0.6)$ the lithium abundance is so low that only upper limits could be determined: $\mathrm{N}(\mathrm{Li})<0.1$.

\subsection{Lithium abundance in the EMP giants}

In a mixing with internal hot layers (like the $\mathrm{H}$ burning layer) we expect that lithium (a very fragile element destroyed as soon as the temperature reaches $2.510^{6} \mathrm{~K}$ ) be almost completely absent at the surface.

In fact, in the "mixed stars" the abundance of lithium is so low that we could only measure upper limits. In Fig. 5 we show the anticorrelation between the lithium abundance and the nitrogen abundance.

\subsection{Mixing and evolution of the star along the giant branch}

If the large scatter of the $\mathrm{C}$ and $\mathrm{N}$ abundances in the EMP giants is explained by a mixing with deep layers, we would expect that this mixing happens at a specific phase of the evolution of the star.

In Fig 6a for our sample of stars, and in Fig. 6b for the less metal-poor sample of Gratton et al. (2000), we have plotted the luminosity of the stars as a function of the temperature. As a comparison, we have added the theoretical isochrones computed by Kim et al. $(2002)$ for $[\mathrm{Fe} / \mathrm{H}]=-2.7$ and -3.7 , (or $[\mathrm{Fe} / \mathrm{H}]=-1.5$ for the sample of Gratton et al., 2000).

In both sample of stars, the mixed giants are represented by open circles and the "unmixed giants" by filled circles. It is clear that the extra-mixing phenomenon begins at a higher luminosity in the extremely metal-poor sample.

Gratton et al. (2000), Denissenkov and VandenBerg (2003) associate this extra-mixing phenomenon in giants to the RGB-bump, and the bump luminosity (LFB) depends strongly on the metallicity of the stars.

Following Denissenkov and VandenBerg $(2003)$ at a metallicity $[\mathrm{Fe} / \mathrm{H}]=-1.5(\mathrm{Z}=0.001$ or $\log \mathrm{Z}=-3.0)$ the $\mathrm{LFB}$ is about at $\log \mathrm{L} / \mathrm{L}_{\odot} \approx 1.95$. They calculate also the $\mathrm{LFB}$ for $\mathrm{Z}=0.002$ and $\mathrm{Z}=0.0005$. Extrapolating these data (Fig.6c), we find for $[\mathrm{Fe} / \mathrm{H}]=-3.1(\log$ $\mathrm{Z}=-4.52)$, LFB at $\approx 2.62$ in good agreement with our observations.

(Note however that recent computations of Meissner and Weiss 2006 find for $[\mathrm{Fe} / \mathrm{H}]=$ $-3.1 \mathrm{LFB}$ at $\approx 2.33$, a value slightly fainter than the observations.)

\section{Behaviour of oxygen, sodium and aluminum in EMP giants}

The abundance of oxygen deduced from the forbidden line at $630.03 \mathrm{~nm}$ is found to be the same in mixed and unmixed giants (Spite et al., 2005: $[\mathrm{O} / \mathrm{Fe}] \approx 0.7$ ) and thus the $\mathrm{ON}$ cycle does not seem to be very efficient in the mixed stars. Let us remark however 

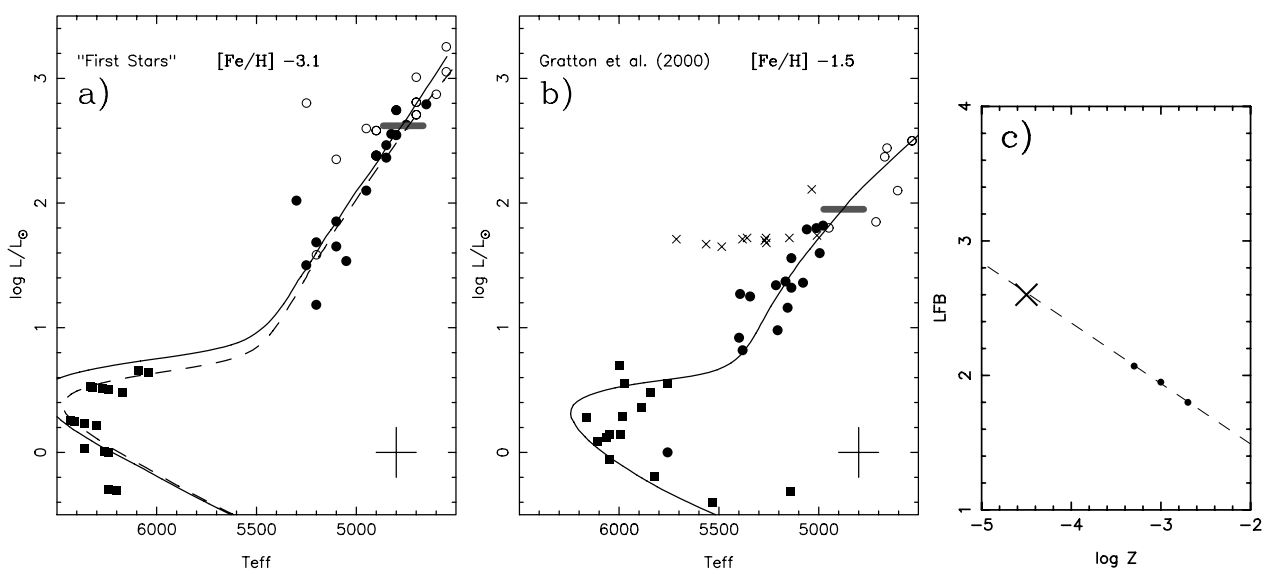

Figure 6. a) HR diagram for our sample of EMP giants $(<[\mathrm{Fe} / \mathrm{H}]\rangle=-3.1)$ and $\mathbf{b})$ for the sample of Gratton et al. (2000) with $<[\mathrm{Fe} / \mathrm{H}]>=-1.5$. The symbols are the same as in Fig 3 . The mixing phenomenon begins at a higher luminosity in the EMP stars, but in both cases this luminosity is close to the luminosity of the bump indicated in the figures by a small thick grey line at $\log \mathrm{L} / \mathrm{L}_{\odot} \approx 2.62$ and 1.95. c) The position of the bump has been deduced from the computations of Denissenkov and VandenBerg (2003): black dots. These computations have been interpolated for $\log \mathrm{Z}=-3.0([\mathrm{Fe} / \mathrm{H}]=-1.5)$ and extrapolated for $\log \mathrm{Z}=-4.52([\mathrm{Fe} / \mathrm{H}]=-3.1)$.
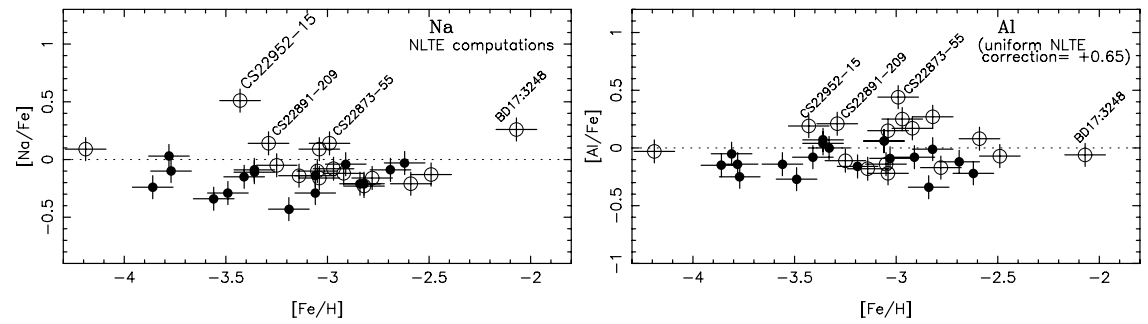

Figure 7. $[\mathrm{Na} / \mathrm{Fe}]$ and $[\mathrm{Al} / \mathrm{Fe}]$ for our sample of EMP giants. The symbols are the same as in Fig. 3. Some mixed stars (and only mixed stars) are Na-rich or/and Al-rich.

that since there are about forty times more oxygen atoms than nitrogen atoms the effect would be much more sensitive on the nitrogen abundance than on the oxygen abundance.

On the contrary the abundances of sodium and aluminum seem, as a mean, to be higher in the mixed giant than in the unmixed giants. In Fig. 7 we have plotted $[\mathrm{Na} / \mathrm{Fe}]$ and $[\mathrm{Al} / \mathrm{Fe}]$ versus $[\mathrm{Fe} / \mathrm{H}]$ for our sample of giants. $[\mathrm{Na} / \mathrm{Fe}]$ deduced from the fit of NLTE profiles of the $\mathrm{D}$ lines has been taken from Andrievsky et al., (2006), and [Al/Fe] from Spite et al. (2006).

In Fig 7 several mixed stars are sodium and/or aluminum rich. We cannot exclude that some of these stars be AGB stars. What is the cause of the $\mathrm{Na} / \mathrm{Al}$ enhancement in some mixed stars? Is it an indication of a deep mixing? of a mass transfer? Let us remark that now after a careful NLTE computation of the sodium abundance (Andrievsky et al., 2006) there is also a very good agreement between the mean abundance of sodium in the turnoff stars and in the unmixed giants and that none of the turnoff stars nor the unmixed giants are Na-rich. 


\section{Conclusion}

As soon as an extremely metal-poor giant reaches the luminosity of the bump its atmosphere is mixed with the H-burning layer and the abundances of the light elements like $\mathrm{C}$ and $\mathrm{N}$ are altered. There is no visible effect on $\mathrm{O}$, but sometimes in the mixed stars the abundances of $\mathrm{Na}$ and $\mathrm{Al}$ are also affected.

\section{References}

Alonso A., Arribas S., Martínez-Roger C., 1999, A\&AS 140, 261

Alvarez R., Plez B., 1998, A\&\&A 330, 1109

Andrievsky S.M., Spite M., Korotin S.A., Spite F., Bonifacio P., et al., 2006 A $\& A$ submitted

Beers T.C., Preston G.W., Shectman S.A., 1992, AJ 103, 1987

Denissenkov P.A., VandenBerg D.A. 2003, ApJ 593, 509

Edvardsson B., Andersen J., Gustafsson B., Lambert D. L., et al., 1993, A\&A 275, 101

Gratton R., Sneden C., Carretta E., Bragaglia A., 2000, A\& $A$ 354, 169

Kim Y.-C., Demarque P., Yi S. K., Alexander D. R., 2002, ApJS, 143, 499

Meissner F., Weiss A., 2006, A\&A 456, 1085

Plez B. Brett J.M., Nordlund Å., 1992, A\&\&A 256, 551

Spite M., Cayrel R., Plez B., Hill V., Spite F., Depagne E., François P., Bonifacio P., Barbuy B., Beers T., Andersen J., Molaro P., Nordström B., Primas F., 2005, A $\& A$ 430, 655

Spite M., Cayrel R., Hill V., Spite F., François P., Plez B., Bonifacio P., Molaro P., Depagne E., Andersen J., Barbuy B., Beers T., Nordström B., Primas F., 2006, A\&A in press, astro-ph/0605056

\section{Discussion}

I.RoxBURGH: Would not O change in H-shell burning, too ?

A.WeIss: Yes, if mixing is deep enough, but for the present effect you only mix to the outer regions of the H-shell. O-Na-anomalies are observed and could be explained by deep mixing. However, they are also observed at the turn-off and then this scenario fails.

M.SPITE: Up to now the O-Na anomaly has been only observed in globular cluster giants, and turnoff stars. Here we are dealing with EMP field giants. In our sample of EMP stars, sodium enrichment has been only observed in some mixed giants but never in unmixed giants or turnoff stars.

C.CHIOsi: The bump traces the location of the $\mathrm{H}$ discontinuity set by the penetration of external convection. Most models do not predict the right luminosity of the observed bump unless some extra mixing is used. As you find that the changes in chemical abundances occur at the bump, can we use the abundances (expected to depend on how deep convection penetrated) to argue for some additional mixing?

M.SPITE: The luminosity of the stars of our sample has been deduced from the gravity (the distances are unknown), and thus it can be affected by a systematic error (Gaia will help). However, it seems established that the sudden change in the $\mathrm{C}$ and $\mathrm{N}$ abundances is linked to a discontinuity in the evolution of the star, and thus, inside the observational errors, to the bump. The abundance changes correspond to some additional mixing. In these EMP stars, and contrary of what has been observed by Gratton in less deficient field stars, this mixing is sometimes very deep since it seems to happen that $\mathrm{Na}$ and sometimes $\mathrm{Al}$ are affected. However we cannot exclude that the Al-rich stars be post helium flash AGB stars, they seem to be located on the left side of the RGB.

Extensive radial velocity monitoring should also constrain the problem (binarity). On the other hand, a NLTE analysis of the aluminum lines is in progress. 